\title{
Nanofriction characteristics of h-BN with electric field induced electrostatic interaction
}

\author{
Kemeng YU ${ }^{1,2}$, Kun ZOU ${ }^{1, *}$, Haojie LANG ${ }^{1}$, Yitian PENG ${ }^{1,2,3, *}$ \\ ${ }^{I}$ Shanghai Collaborative Innovation Center for High Performance Fiber Composites, Donghua University, Shanghai 201620, \\ China \\ ${ }^{2}$ College of Mechanical Engineering, Donghua University, Shanghai 201620, China \\ ${ }^{3}$ Engineering Research Center of Advanced Textile Machinery, Donghua University, Shanghai 201620, China \\ Received: 19 January 2020 / Revised: 12 June 2020 / Accepted: 11 July 2020 \\ (C) The author(s) 2020.
}

\begin{abstract}
The nanofriction properties of hexagonal boron nitride (h-BN) are vital for its application as a substrate for graphene devices and solid lubricants in micro- and nano-electromechanical devices. In this work, the nanofriction characteristics of $\mathrm{h}-\mathrm{BN}$ on $\mathrm{Si} / \mathrm{SiO}_{2}$ substrates with a bias voltage are explored using a conductive atomic force microscopy (AFM) tip sliding on the h-BN surface under different substrate bias voltages. The results show that the nanofriction on h-BN increases with an increase in the applied bias difference $\left(V_{\mathrm{t}-\mathrm{s}}\right)$ between the conductive tip and the substrate. The nanofriction under negative $V_{\mathrm{t}-\mathrm{s}}$ is larger than that under positive $V_{\mathrm{t}-\mathrm{s}}$. The variation in nanofriction is relevant to the electrostatic interaction caused by the charging effect. The electrostatic force between opposite charges localized on the conductive tip and at the $\mathrm{SiO}_{2} / \mathrm{Si}$ interface increases with an increase in $V_{\mathrm{t}-\mathrm{s}}$. Owing to the characteristics of p-type silicon, a positive $V_{\mathrm{t}-\mathrm{s}}$ will first cause depletion of majority carriers, which results in a difference of nanofriction under positive and negative $V_{\mathrm{t}-\mathrm{s}}$. Our findings provide an approach for manipulating the nanofriction of 2D insulating material surfaces through an applied electric field, and are helpful for designing a substrate for graphene devices.
\end{abstract}

Keywords: nanofriction; electrostatic interaction; bias voltage; h-BN; atomic force microscopy

\section{Introduction}

Electric fields play a critical role in substrate materials and interface lubricating materials. Graphene devices supported by different substrates are highly disordered, and the geometry of the substrate imposes severe limitations on the function of graphene devices [1, 2]. The quantum anomalous Hall effect in graphene and its bilayer was first observed in 2013 [3, 4], and research regarding the effect of substrates on graphene devices continues. The carrier mobility of $\mathrm{SiO}_{2}$-supported graphene devices is limited by scattering from charged surface states and impurities
[5-9] and substrate surface roughness [10-12]. For $\mathrm{Si}$, the electronic contributions to friction in silicon $\mathrm{p}-\mathrm{n}$ junctions have been studied by Park et al. [13, 14], who reported that the bias influenced the friction of the silicon $\mathrm{p}-\mathrm{n}$ junction by adjusting the state of the electronic surface. Owing to the electrostatic force, the bias voltage results in a significant increase in the friction force at an applied electric field [15]. However, there is still a problem with the surface effects of substrate substitutes for graphene devices [16-18]. Contact electrification friction is a universal phenomenon of electrostatic interaction in the process of contact sliding between two materials surfaces [19,

* Corresponding authors: Kun ZOU, E-mail: kouz@dhu.edu.cn; Yitian PENG, yitianpeng@dhu.edu.cn 
20]. It plays an important role in electrostatic-driven micromotors at the electrified interfaces [21, 22] and in electric motors [23]. The electronic control of the friction force at the atomic scale is related to the energy dissipation in mechanical energy via electron-hole pair creation. Dayo et al. [24] explored the electronic contributions to friction using a quartz crystal microbalance. Meyer's work in non-contact atomic force microscopy (AFM) proved that photonic friction is the main dissipative channel below the critical temperature [25], and Park et al. [26, 27] studied the contribution of photonic and electronic dissipation to friction. Therefore, understanding the connection between the interfacial electrostatic interaction and nanofriction is of paramount importance in the design of graphene devices and solid lubricants.

Hexagonal boron nitride (h-BN) is a single atomic layer consisting of an $\mathrm{sp}^{2}$ hybridized boron and a nitrogen compound in a honeycomb lattice [28-30]. The h-BN serves as a semiconductor with a wide bandgap near $6 \mathrm{eV}$ that possesses much higher chemical and thermal stability [31-33]. Therefore, it can be applied to the dielectric layer and deep ultraviolet emitter [34, 35]. The atomically planar surface of h-BN should suppress rippling in graphene $[10,36]$. Graphene devices on singlecrystal h-BN substrates have higher quality and less intrinsic doping compared to $\mathrm{SiO}_{2}$-supported devices [37]. The friction and adhesion of the electrical contact sliding interface on h-BN are critical to the performance, life, and reliability of graphene nanoelectronics devices.

The potential difference between AFM tips with different conductivities and samples was applied to study bias-dependent friction using conductive AFM [38, 39]. In addition, Fann et al. [40] reported electron dynamics and thermalization in metals and semiconductors on timescales, providing an exciting possibility to control the nanofriction of h-BN by using charges. Here, the nanoscale friction and adhesion characteristics of h-BN were studied by applying an independent bias on a conductive AFM tip and substrate in an ambient environment. With many recorded force-distance curves of h-BN, the bias-dependent nanofriction of h-BN through electrostatic interactions under different electric fields was studied. The friction and adhesion bias-dependent mechanism of h-BN at the electrified sliding interface are presented.

\section{Experimental}

All nanofriction and adhesion measurements were performed on a beam deflection AFM (MFP-3D, Asylum Research, USA) under an ambient environment (63\% relative humidity and room temperature of $\left.27 \pm 2{ }^{\circ} \mathrm{C}\right)$. The h-BN sample with an intact structure was obtained through the mechanical exfoliation method from h-BN powder [37, 41] deposited onto a p-type $\mathrm{Si}$ substrate with $300-\mathrm{nm} \mathrm{SiO}_{2}$, and the bottom surface was coated with 60-nm Au. The substrate was ultrasonically cleaned in acetone, isopropyl alcohol, and deionized water for $10 \mathrm{~min}$ each. Optical microscopy and AFM were utilized to determine the location and thickness of the h-BN on the $\mathrm{SiO}_{2} / \mathrm{Si} / \mathrm{Au}$ substrate.

Commercial silicon cantilevers were used in the tapping mode and contact mode to obtain the AFM morphology and friction image. Commercial silicon conductive probes with 25-nm PtIr coating (EFM, NanoWorld, Switzerland) were used throughout the experiments. With spring constants of the cantilevers ranging from 1.2 to $5.5 \mathrm{~N} / \mathrm{m}$, a noncontact method can be used to determine the spring constant of the cantilever [42]. The resonant frequency was $75 \mathrm{kHz}$. The method proposed by Green et al. was used to determine the lateral force stiffness of the cantilever [43]. The normal cantilever value is $0 \mathrm{nN}$ when there is no direct contact between the tip and the sample surface during the measurement. The positive and negative values of force are compressive forces and adhesion, respectively. As the tip slid along the h-BN surface, the twisting signal from the cantilever twists was used to measure lateral forces. Friction was calculated by averaging the difference between the lateral forces while the tip slid forward and backward. In the surrounding environment, all experimental measurements can be obtained with different conductive tips and h-BN samples.

A method was used to control nanofriction with a bias as follows. The tip was pressed against the surface of h-BN when the substrate bias was pre- 
determined. For this purpose, an image of the h-BN surface can be acquired. Subsequently, under a normal load of $60 \mathrm{nN}$, the tip bias was linearly increased or decreased until a predetermined bias was obtained, and then the tip jumped out of contact with the surface. The force-distance curves under different tip biases and substrate biases were measured. Scanning Kelvin probe microscopy (SKPM) was used to measure the potential difference between the tip and $\mathrm{h}-\mathrm{BN}$ at ambient conditions. The conductive tip still exhibited good electrical conductivity during the AFM measurements (Fig. S1 in the Electronic Supplementary Material (ESM)). The scan rate was fixed at $1 \mathrm{~Hz}$ for all measurements, and the scan size of the experiments was $500 \mathrm{~nm} \times 500 \mathrm{~nm}$.

\section{Results and discussion}

All electrostatic nanofriction and adhesion measurements were conducted on h-BN under various electric fields. Figure 1(a) shows a schematic diagram of the experimental setup, where h-BN is deposited on a $\mathrm{Si}$ substrate with a $300-\mathrm{nm} \mathrm{SiO}_{2}$ layer, and the bottom surface is coated with 60-nm Au. The conductive tip and substrate were applied with positive or negative bias. Figures $1(\mathrm{~b})$ and $1(\mathrm{c})$ show the topography of h-BN measured by AFM. The inset shows the height profile of h-BN; the white line representing the height of the h-BN sheet is $3.3 \mathrm{~nm}$.

Figure 2 shows the friction as a function of the tip bias under different substrate biases. It can be seen that the friction increases with increasing applied tip bias regardless of whether the tip bias is positive or negative. In Fig. 2(a), when the substrate bias is $0 \mathrm{~V}$, as the tip bias increases from 0 to $5 \mathrm{~V}$ in the opposite way, the friction increases to $3.1 \mathrm{nN}$ from the previous value of $1.8 \mathrm{nN}$. Similarly, the friction increases from 1.7 to $1.9 \mathrm{nN}$ as the tip bias increases from 0 to $5 \mathrm{~V}$. Figure $2(\mathrm{~b})$ shows that the friction on h-BN increases from 1.77 to $3.29 \mathrm{nN}$ as the tip bias increases linearly from $0 \mathrm{~V}$ to approximately $5 \mathrm{~V}$ in the opposite direction under a substrate bias of $5 \mathrm{~V}$. Likewise, the friction on h-BN increases from 1.7 to $2.8 \mathrm{nN}$ as the tip bias increases linearly from $0 \mathrm{~V}$ to nearly $5 \mathrm{~V}$ under a substrate bias of $-5 \mathrm{~V}$. Figures 2(c) and 2(d) show a plot of the friction versus the friction cycle, in which the effect of the bias on the friction is observed, where $V_{\mathrm{t}}$ is the conductive bias. Figures S2(a)-S2(d) (ESM) show the nanofriction images in Figs. 2(c) and 2(d). During the entire friction experiment, the normal load remained at $60 \mathrm{nN}$.

The friction changed further when a bias of the same polarity was applied to both the conductive tip and the substrate. In Fig. 3(a), when the substrate bias is $5 \mathrm{~V}$, the friction on h-BN decreases from 2.97 to $1.75 \mathrm{nN}$ as the tip bias increases linearly from $0 \mathrm{~V}$ to approximately $5 \mathrm{~V}$. Similarly, when the substrate bias is $-5 \mathrm{~V}$, the friction on h-BN decreases from 2.0 to $1.67 \mathrm{nN}$ as the tip bias increases linearly from $0 \mathrm{~V}$ to approximately $5 \mathrm{~V}$ in the opposite way. Figure 3(b) shows that the friction decreases with an increase in the tip bias until the tip bias approaches the contact potential difference (CPD) between the Pt/Ir-coated tip and the sample surface. Beyond the bias, the friction increases with an increase in the tip bias. In Fig. 3(b), the friction on h-BN decreases from 2.7 to $1.7 \mathrm{nN}$ and then increases from 1.7 to $1.9 \mathrm{nN}$ as the tip bias increases horizontally from $0 \mathrm{~V}$ to approximately $8 \mathrm{~V}$
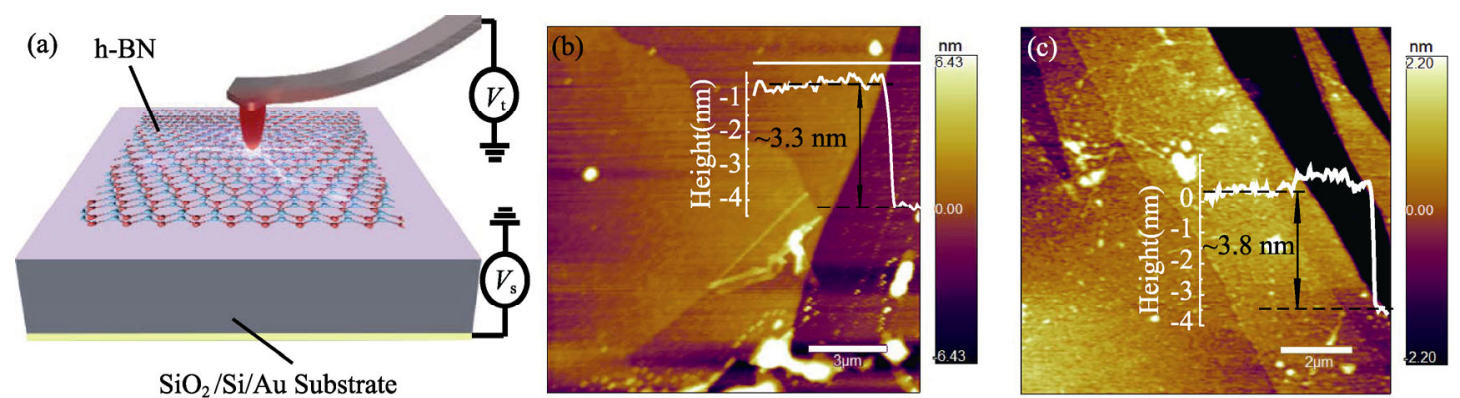

Fig. 1 (a) Schematic diagram of the conductive AFM experimental setup. (b, c) Topography images of h-BN. The inset shows the height profile of h-BN. 

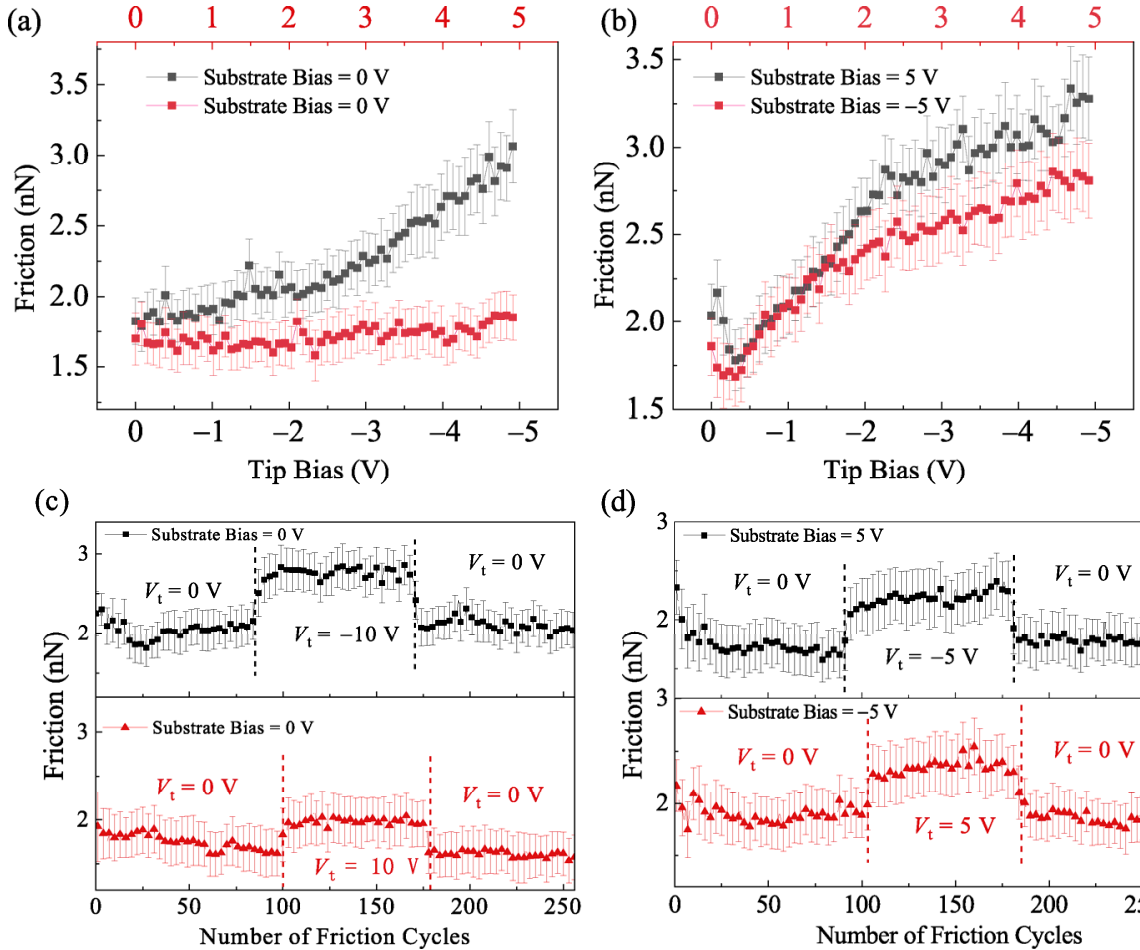

(d)

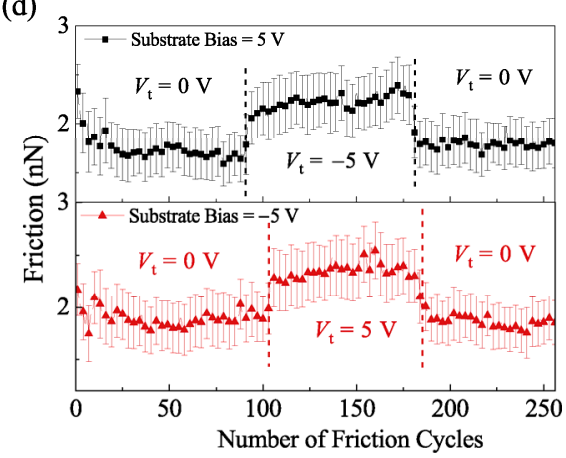

Fig. 2 Friction as a function of the tip bias under different substrate biases. (a) The substrate bias is $0 \mathrm{~V}$. (b) The substrate bias is 5 and $-5 \mathrm{~V}$, respectively. Variation of friction versus the number of friction cycles before and after several bias voltages were applied, respectively. (c) $V_{\mathrm{t}}=-10$ and $10 \mathrm{~V}$. (d) $V_{\mathrm{t}}=-5$ and $5 \mathrm{~V}$. The normal force is $60 \mathrm{nN}$.

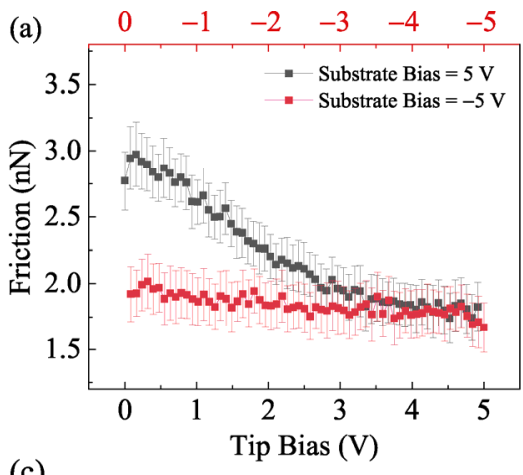

(c)

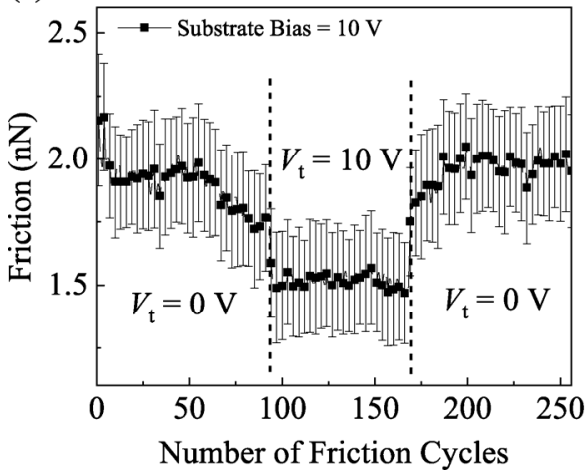

(b)

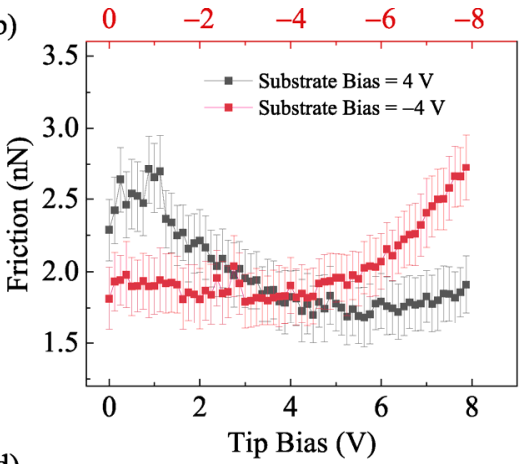

(d)

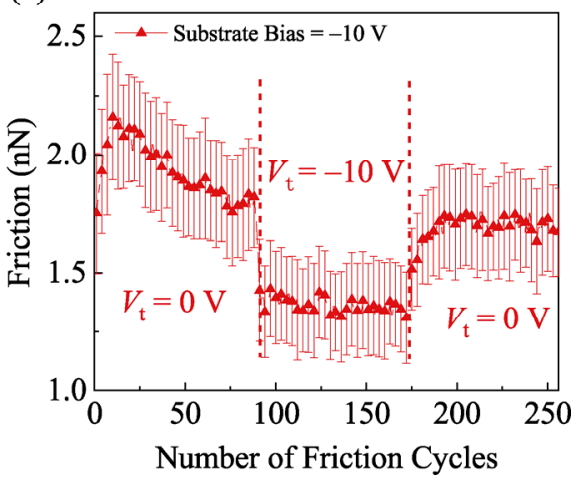

Fig. 3 Variations of friction as a function of the tip bias. (a) Substrate bias of 5 and -5 V, respectively. (b) Substrate bias of 4 and $-4 \mathrm{~V}$, respectively. Variation of friction versus the number of friction cycles before and after several bias voltages were applied, respectively. (c) $V_{\mathrm{t}}=10 \mathrm{~V}$ and (d) $V_{\mathrm{t}}=-10 \mathrm{~V}$. The substrate bias is fixed, and the normal force is $60 \mathrm{nN}$. 
under a substrate bias of $4 \mathrm{~V}$. The friction on h-BN decreases from 2.0 to $1.8 \mathrm{nN}$ and then increases from 1.8 to $2.7 \mathrm{nN}$ as the tip bias increases horizontally from $0 \mathrm{~V}$ to nearly $8 \mathrm{~V}$ in the opposite direction under a substrate bias of $-4 \mathrm{~V}$. It can be seen in Fig. 3 that h-BN has higher friction when a positive bias is applied to the substrate than for a negative bias. Figures 3(c) and 3(d) show a plot of friction versus the friction cycle, in which the effect of the bias on the friction is observed. Figures S2(e) and S2(f) (ESM) show the nanofriction images in Figs. 3(c) and 3(d). During the entire friction experiment, the normal load remained at $60 \mathrm{nN}$.

According to Amonton's law [44], the friction force $\left(F_{\mathrm{f}}\right)$ can be expressed as

$$
F_{\mathrm{f}}=\mu\left(F_{\mathrm{N}}+F_{\mathrm{ad}}\right)
$$

where $\mu$ is the friction coefficient, $F_{\mathrm{N}}$ is the normal force applied to the probe, and $F_{\text {ad }}$ is the adhesion force between the tip and the sample.

When a bias is applied to the conductive tip and the substrate, the change in friction mainly results from the change in the adhesion between the tip and the h-BN sample [45]. To quantify the electric

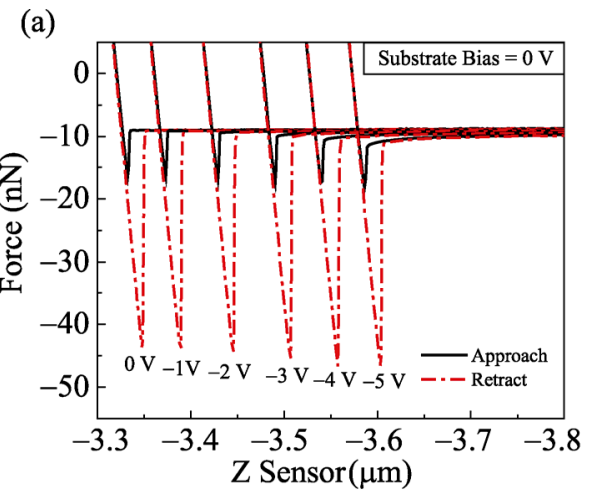

(c)

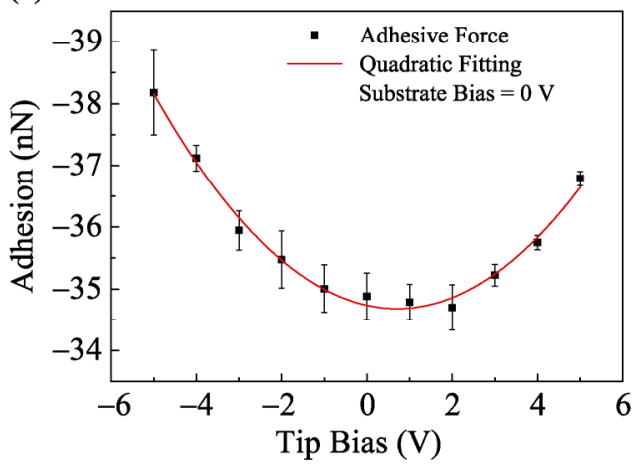

field influence, the force-distance curves under different tip biases were measured under a fixed substrate bias. Figure 4(a) displays the force-distance curve with different tip biases ranging from 0 to $-5 \mathrm{~V}$ under a substrate bias of $0 \mathrm{~V}$. The solid black lines represent the interaction as the tip approaches $\mathrm{h}-\mathrm{BN}$, and the dotted red lines show the interaction as the tip retracts from the h-BN. A similar trend appears in Fig. 4(b) with different tip biases, ranging from 0 to $5 \mathrm{~V}$, under a substrate bias of $0 \mathrm{~V}$. The fitting curve between adhesion and the tip bias is shown in Fig. 4(c) to determine the influence of electrostatic force on adhesion.

The change in adhesion was recorded when a bias of the opposite polarity was applied to both the conductive tip and the substrate. Figure 5(a) shows the adhesion as a function of tip bias under a substrate bias of 5 and $-5 \mathrm{~V}$, respectively. The adhesion between the tip and the h-BN surface increases gradually with the tip bias approaching the substrate bias, regardless of whether a positive bias or negative bias is applied to the conductive tip. Figure 5(a) shows the difference in adhesion between the negative and positive bias at the same bias.

(b)

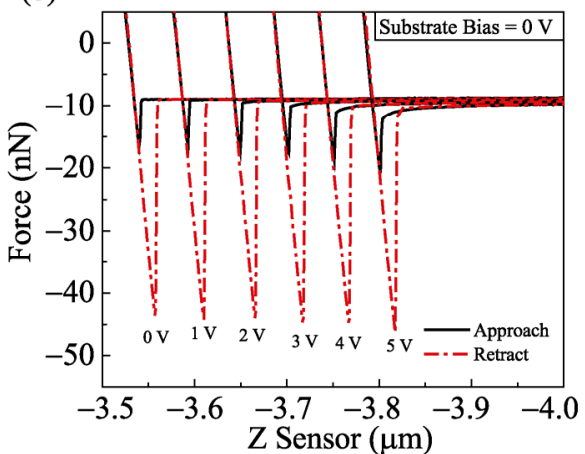

(d)

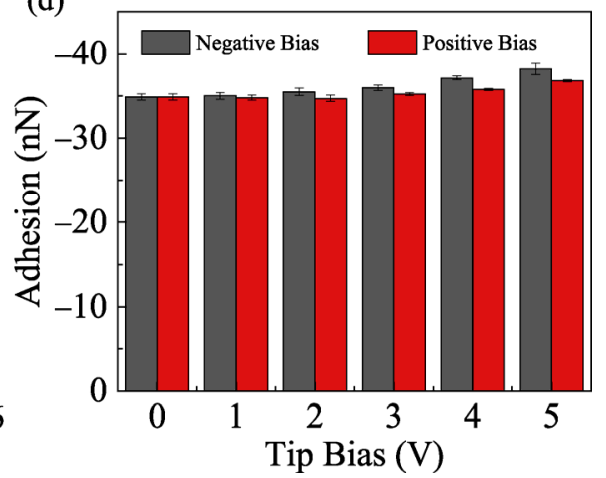

Fig. 4 Force-distance curve under different tip biases. (a) Positive bias and (b) negative bias. (c) Adhesive force as a function of tip bias voltage. (d) The difference in adhesion between negative and positive bias. 

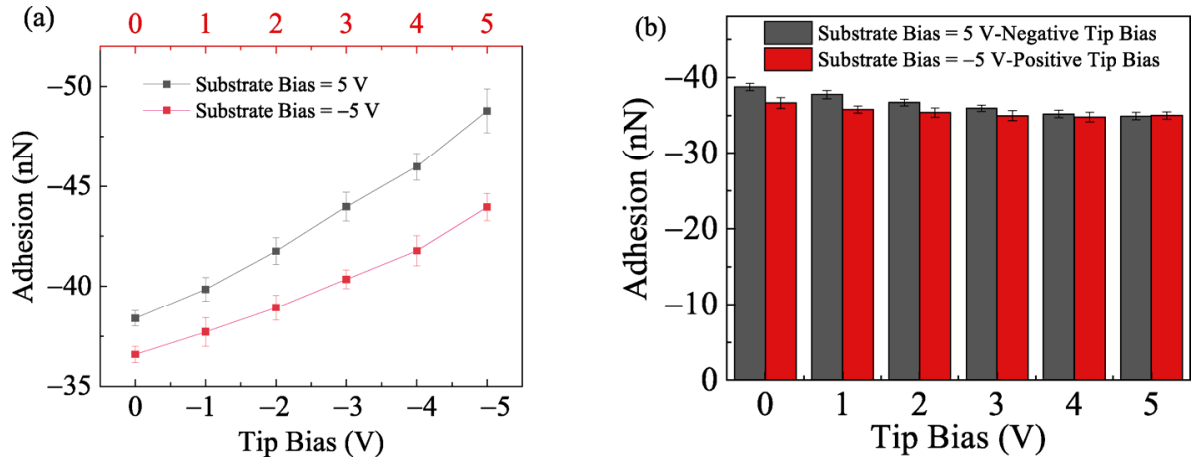

Fig. 5 (a) Adhesion as a function of tip bias when a bias of the opposite polarity is applied to both the conductive tip and the substrate. (b) The difference in adhesion between negative and positive bias at the same bias.

The change in adhesion was recorded when a bias of the same polarity was applied to both the substrate and the conductive tip. Figure 6(a) shows the adhesion as a function of tip bias under a substrate bias of 5 and $-5 \mathrm{~V}$, respectively. The adhesion between the tip and the h-BN surface decreases gradually as the tip bias approaches the substrate bias. In Fig. 6(b), the adhesion decreases with an increase in the tip bias until the tip bias approaches the CPD between the tip and the sample surface; beyond the bias, the adhesion increases with an increase in the tip bias.

Considering the effect of the contact area, the relationship between the contact area and the load usually agrees with the Derjaguin-Müller-Toporov (DMT) models [46]. The friction-load curves at different biases are shown in Fig. 7. Figure 7(a) shows the friction as a function of load with no bias. Fitting with the DMT model was performed. The DMT model was used to calculate the contact

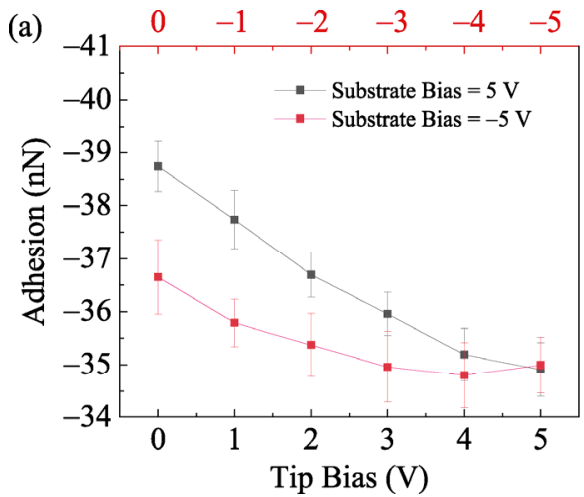

area. The friction-load curves of the tip under a bias of +1 and $-1 \mathrm{~V}$ are shown in Figs. 7(b) and 7(c), respectively, along with the fit to the DMT models.

The relationship between friction and load can be expressed as

$$
\frac{F}{F_{0}}\left(\frac{\alpha+\sqrt{1-\frac{L^{2}}{L_{\mathrm{c}}}}}{1+\alpha}\right)^{4 / 3}
$$

where $F_{0}$ is friction at zero load, and $L_{c}$ is the pull-off force. When the DMT model is adopted, $\alpha=0$. In the DMT model, $L_{\mathrm{c}}$ is given by

$$
L_{\mathrm{c}}=-2 \pi \gamma R
$$

where $R$ is the tip radius, and $\gamma$ is the adhesion work. The contact radius $a_{0}$ at zero load is calculated according to the DMT model as

$$
a_{0}=\left(\frac{2 \pi \gamma R^{2}}{k}\right)^{1 / 3}
$$

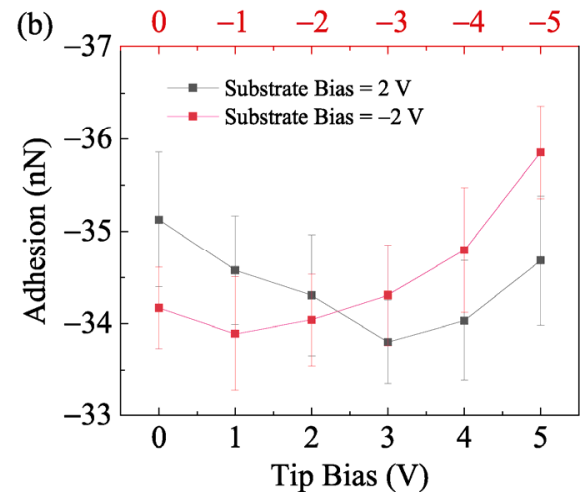

Fig. 6 Adhesion as a function of the tip bias when a bias of the same polarity is applied to both the substrate and the conductive tip. (a) Under a substrate bias of 5 and $-5 \mathrm{~V}$, respectively. (b) Under a substrate bias of 2 and $-2 \mathrm{~V}$, respectively. 
where $k$ is the combined elastic modulus of the tip and the sample, given by

$$
k=\frac{4}{3}\left(\frac{1-v_{1}^{2}}{E_{1}}+\frac{1-v_{2}^{2}}{E_{2}}\right)^{-1}
$$

where $E$ and $v$ are the elasticity modulus and Poisson's ratio, respectively. The tip radius was approximately $30 \mathrm{~nm}$ (PtIr coated conductive tip, Nanosensors). The thickness of h-BN is approximately $3 \mathrm{~nm}$, the elasticity modulus $\left(E_{1}=856 \mathrm{GPa}\right)$ and Poisson's ratio $\left(v_{1}=0.211\right)$ were used in the calculation [47]. In addition, the elasticity modulus $\left(E_{2}=190\right.$ GPa $)$ and Poisson's ratio $\left(v_{2}=0.30\right)$ for PtIr coating on the tip were used [48].

Combining Eqs. (2)-(5), the contact area can be calculated, and the variation of the contact area with the tip bias is shown in Fig. 7(d). When the values of the bias were the same, the contact radius and contact area measured with a positively-biased tip were lower than those measured by a negativelybiased tip. The application of bias changes the contact area between the tip and h-BN, and increases the real contact area to a small extent, which means that the electrostatic adhesion could be the main factor inducing friction enhancement.

When a bias difference is applied to the conductive tip and the substrate, the electronic bands bend (in energy) at the oxide-semiconductor interface. The degree of bending depends on the magnitude and direction of the bias difference. Figure 8(a) shows a schematic diagram of the experimental setup, illustrating the application method of the bias voltage on the conductive tip and the substrate. During the friction experiment, the conductive tip is always in contact with the surface of h-BN. The bias difference between the conductive tip and the substrate denoted as $V_{\mathrm{t}-\mathrm{s}}$, which is given by

$$
V_{\mathrm{t}-\mathrm{s}}=V_{\mathrm{t}}-V_{\mathrm{s}}
$$

where $V_{\mathrm{t}}$ denotes the bias of the conductive tip, and $V_{s}$ is the bias of the substrate.

The experimental device diagram shown in Fig. 8(a) is equivalent to a metal-oxide-p-type semiconductor (MOS) capacitor. Figure 8(b) shows the energy band diagram of the MOS structure without applied bias; there are no trapping charges in the oxide. The flat band voltage is simply equal to the work function difference between the conductive tip and silicon,
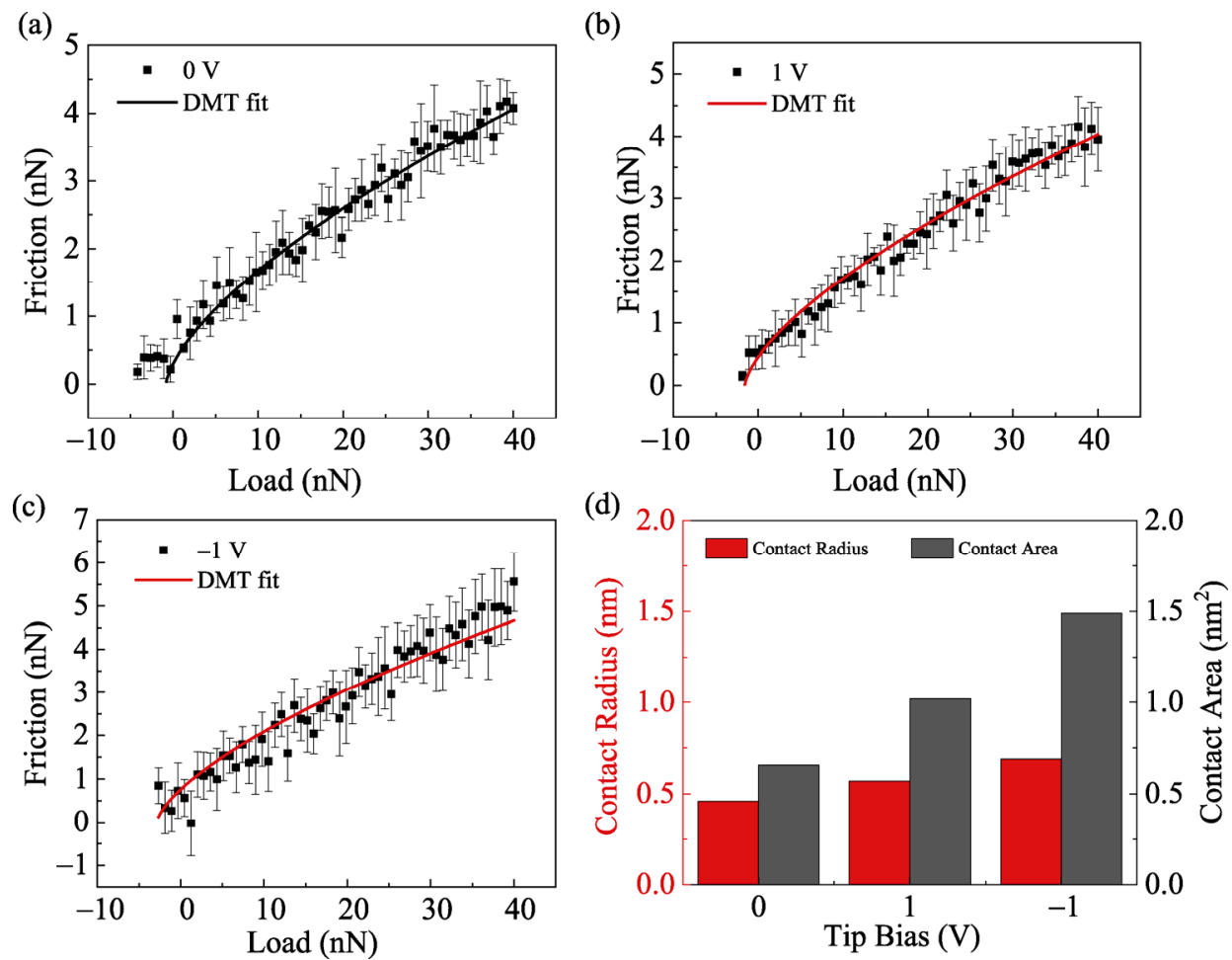

Fig. 7 Friction force as a function of load with (a) $0 \mathrm{~V}$, (b) $\pm 1 \mathrm{~V}$, and (c) $\pm 2 \mathrm{~V}$ tip bias. The solid lines are fits to the DMT models. (d) Contact area and contact radius as a function of tip bias. 
where $e \phi_{\mathrm{m}}$ is the conductive tip Fermi energy with respect to the vacuum level $E_{\mathrm{vcc}}, \chi$ is the electron affinity for the silicon, $E_{c}$ is the conduction band edge, and $E_{\mathrm{v}}$ is the valence band edge. For p-type silicon, the Fermi energy $E_{\mathrm{Fi}}$ is above the intrinsic Fermi energy $E_{\mathrm{F}}$. When the bias difference is applied to both ends of the MOS, it is equivalent to the fact that both sides of the conductive tip and p-type silicon are charged, the charges on both sides are equal, and the signs of the charges are opposite. Owing to the high electron density of the conductive tip, the charge distribution is within the thickness of approximately one atomic layer near the surface. For p-type silicon, the carrier density is much lower than that of the conductive tip, and the charge is only distributed in the surface layer of a certain thickness. When $V_{\mathrm{t}-\mathrm{s}}$ is a negative bias difference, the electronic bands' bend, and most carrier stacking states are shown in Fig. 8(c). The accumulated holes are mainly distributed in the thin layer near the surface. During the charging process, negative charges appear on one side of the conductive tip, while positive charges will appear on the other side
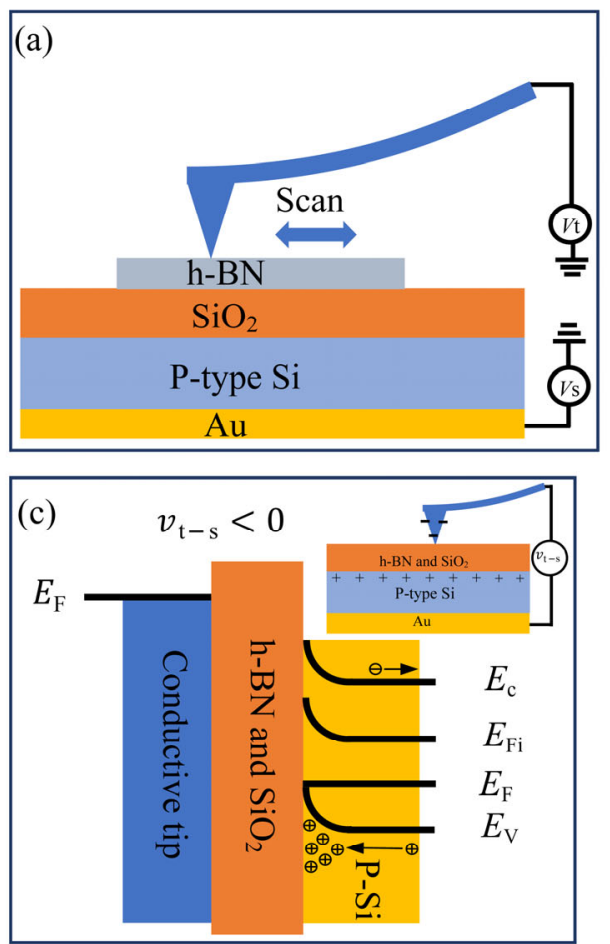

of the semiconductor, and the electric field appears in the space charge area of the semiconductor. When $V_{\mathrm{t}-\mathrm{s}}$ is negative, the electronic bands bend and most carrier stacking states are shown in Fig. 8(d); the charge distribution and electric field direction are opposite to those in Fig. 8(c). The hole concentration near the surface is much smaller than that in the body, and most carriers near the surface are depleted. With an increase in $V_{\mathrm{t}-\mathrm{s}}$, the increase in charges and the enhancement of the electric field in the space charge area will lead to the inversion of minority carriers. The electronic bands are more curved. The p-type semiconductor near the surface shows the characteristics of n-type semiconductors, and the electrons will accumulate in the electron inversion layer. The inset shows the charge distribution. These charging effects lead to electrostatic interactions.

To understand the proposed theoretical model, the variation of the adhesion experiment is analyzed as follows. The adhesion $\left(F_{\text {ad }}\right)$ can be expressed as

$$
F_{\mathrm{ad}}=F_{\mathrm{vdw}}+F_{\mathrm{e}}+F_{\mathrm{me}}+F_{\mathrm{ch}}
$$
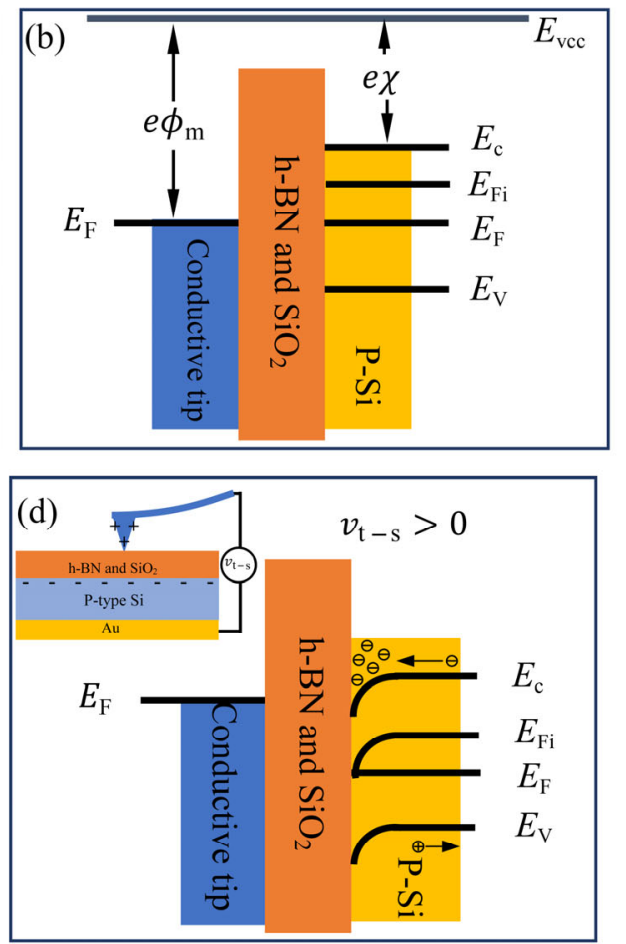

Fig. 8 (a) Schematic diagram of the experimental setup, (b) the energy band diagram of the experimental structure without applied bias, (c) the energy band bending diagram under negative $V_{\mathrm{t}-\mathrm{s}}$, and (d) the energy band bending diagram under positive $V_{\mathrm{t}-\mathrm{s}}$. The insets in (c) and (d) show the charge distribution. 
where $F_{\mathrm{vdw}}$ is the van der Waals force, which is a distance-dependent interaction between atoms or molecules. Hence, $F_{\text {vdw }}$ does not change with the tip bias. The h-BN has a smooth surface that is relatively free of dangling bonds, which ignores the chemical bonding force $\left(F_{\mathrm{ch}}\right)$. The term $F_{\mathrm{e}}$ is the electrostatic force generated when the bias is applied to the tip, which must be taken into consideration in the calculation of $F_{\text {ad }}$. The bias applied to the tip makes polar water molecules form a water meniscus on the surface of the sample $[49,50]$. Thus, $F_{\text {me }}$ may influence adhesion.

The change in adhesion with the bias of the tip was recorded when the substrate was grounded. To determine the influencing factors of adhesion during application of $V_{\mathrm{t}-\mathrm{s}}$, the quadratic fitting of adhesion was introduced. As shown in Fig. 4(c), the adhesion is proportional to the square of the bias, which satisfies the effect of the electrostatic force on the adhesion and corresponds to Coulomb's law:

$$
F_{\mathrm{e}}=k \frac{q_{1} q_{2}}{r^{2}}
$$

where $k$ is the Coulomb constant, $q_{1}$ is the surface charge on the tip surface, $r$ is the distance between the charge, and $q_{2}$ is the opposite charge at the $\mathrm{Si} / \mathrm{SiO}_{2}$ interface [51], which can be explained by the theoretical model in Fig. 8. The fitting result shown in Fig. 4(c) is

$$
F_{\text {ad }}=-0.12\left(V_{\mathrm{t}}-0.71\right)^{2}-34.68
$$

where $F_{\text {ad }}$ is the adhesion, and $V_{t}$ is the tip bias. When $V_{\mathrm{t}}=0.71 \mathrm{~V}$, the adhesion reaches the minimum value. The inherent potential difference, arising from the work function difference between the conductive tip and the h-BN sample, can lead to electrostatic forces [52-54]. A positive $V_{\mathrm{t}-\mathrm{s}}$ must first counteract the potential difference to adjust the Fermi level of the two materials to reach the same level, so that the adhesion reaches the minimum value(Fig. S3 in the ESM). This is the depletion state of the majority carriers. In Fig. 2(a), the difference in friction between +4.7 and $-3.3 \mathrm{~V}$ remains, even after the CPD is overcome, which could be attributed to the accumulation and depletion of major carriers [13]. This is why the adhesion of h-BN under positive $V_{\mathrm{t}-\mathrm{s}}$ is less than that under negative $V_{\mathrm{t}-\mathrm{s}}$.

Next, the change in adhesion with the bias of the tip is discussed when the bias of the opposite or the same polarity is applied to both the substrate and the conductive tip. The experimental results also satisfy the theoretical model proposed in Fig. 8; that is, the variation of adhesion is relevant to the electrostatic interaction caused by the charging effect. When $V_{\mathrm{t}-\mathrm{s}}$ exists, similar to charging a capacitor, the opposite charge is distributed on the conductive tip surface and at the $\mathrm{Si} / \mathrm{SiO}_{2}$ interface. Therefore, both positive $V_{\mathrm{t}-\mathrm{s}}$ and negative $V_{\mathrm{t}-\mathrm{s}}$ can induce electrostatic attraction between the conductive tip and the substrate, thus changing the normal load of the conductive tip. The difference in adhesion between negative $V_{\mathrm{t}-\mathrm{s}}$ and positive $V_{\mathrm{t}-\mathrm{s}}$ can also be explained by the theoretical models in Fig. 8. Since $\mathrm{h}-\mathrm{BN}$ is hydrophobic, the influence of the water meniscus on adhesion can be ignored [55]. The experimental results are consistent with the theoretical model proposed in Fig. 8.

\section{Conclusions}

The nanofriction of h-BN can be tuned by applying $V_{\mathrm{t}-\mathrm{s}}$ to both the substrate and the conductive tip. Similar to charging a capacitor, the opposite charge is distributed on the conductive tip surface and at the $\mathrm{Si} / \mathrm{SiO}_{2}$ interface. The electrostatic attraction generated by the charging effect leads to a change in the normal load; thereby changing the friction and adhesion of the h-BN. Owing to the characteristics of p-type silicon, a positive $V_{\mathrm{t}-\mathrm{s}}$ will first cause depletion of majority carriers, which results in a difference of electrostatic friction and adhesion under positive and negative $V_{\mathrm{t}-\mathrm{s}}$. The mechanism studied here may provide a new approach for controlling the nanofriction of 2D insulating material surfaces at the nanoscale of contact electrification, and it is crucial for its application as a substrate for graphene devices in micro- and nano-electromechanical devices.

\section{Acknowledgments}

This work was supported by the National Natural Science Foundation of China (51675097, U1632128, 
and 51775105), the Natural Science Foundation of Shanghai (17ZR1400700), and the Fundamental Research Funds for the Central Universities ad DHU Distinguished Young Professor Program.

\section{Electronic Supplementary Material Supplementary} material is available in the online version of this article at https://doi.org/10.1007/s40544-020-0432-x.

Open Access This article is licensed under a Creative Commons Attribution 4.0 International License, which permits use, sharing, adaptation, distribution and reproduction in any medium or format, as long as you give appropriate credit to the original author(s) and the source, provide a link to the Creative Commons licence, and indicate if changes were made.

The images or other third party material in this article are included in the article's Creative Commons licence, unless indicated otherwise in a credit line to the material. If material is not included in the article's Creative Commons licence and your intended use is not permitted by statutory regulation or exceeds the permitted use, you will need to obtain permission directly from the copyright holder.

To view a copy of this licence, visit http://creativecommons.org/licenses/by/4.0/.

\section{References}

[1] Bolotin K I, Sikes K J, Hone J, Stormer H L, Kim P. Temperature-dependent transport in suspended graphene. Phys Rev Lett 101(9): 096802 (2008)

[2] Du X, Skachko I, Barker A, Andrei E Y. Approaching ballistic transport in suspended graphene. Nat Nanotechnol 3(8): 491-495 (2008)

[3] Castro Neto A H, Guinea F, Peres N M R, Novoselov K $\mathrm{S}$, Geim A K. The electronic properties of graphene. Rev Mod Phys 81(1): 109-162 (2009)

[4] Geim A K, Novoselov K S. The rise of graphene. Nanosci Technol 6(3): 183-191 (2007)

[5] Ando T. Screening effect and impurity scattering in monolayer graphene. J Phys Soc Jpn 75(7): 074716 (2006)

[6] Nomura K, MacDonald A H. Quantum transport of massless Dirac fermions. Phys Rev Lett 98(7): 076602 (2007)

[7] Hwang E H, Adam S, Das Sarma S. Carrier transport in two-dimensional graphene layers. Phys Rev Lett 98(18): 186806 (2007)
[8] Adam S, Hwang E H, Galitski V M, Das Sarma S. A self-consistent theory for graphene transport. Proc Natl Acad Sci USA 104(47): 18392-18397 (2007)

[9] Chen J H, Jang C, Xiao S D, Ishigami M, Fuhrer M S. Intrinsic and extrinsic performance limits of graphene devices on $\mathrm{SiO}_{2}$. Nat Nanotechnol 3(4): 206-209 (2008)

[10] Ishigami $M$, Chen $J ~ H$, Cullen $W$ G, Fuhrer $M \quad S$, Williams E D. Atomic structure of graphene on $\mathrm{SiO}_{2}$. Nano Lett 7(6): 1643-1648 (2007)

[11] Katsnelson M I, Geim A K. Electron scattering on microscopic corrugations in graphene. Philos Trans A Math Phys Eng Sci 366(1863): 195-204 (2008)

[12] Morozov S V, Novoselov K S, Katsnelson M I, Schedin F, Elias D C, Jaszczak J A, Geim A K. Giant intrinsic carrier mobilities in graphene and its bilayer. Phys Rev Lett 100(1): 016602 (2008)

[13] Park J Y, Ogletree D F, Thiel P A, Salmeron M. Electronic control of friction in silicon pn junctions. Science 313(5784): 186 (2006)

[14] Park J Y, Qi Y B, Ogletree D F, Thiel P A, Salmeron M. Influence of carrier density on the friction properties of silicon pn junctions. Phys Rev B 76(6): 064108 (2007)

[15] Jiang Y, Yue L L, Yan B S, Liu X, Yang X F, Tai G A, Song J. Electric control of friction on silicon studied by atomic force microscope. Nano 10(3): 1550038 (2015)

[16] Ponomarenko L A, Yang R, Mohiuddin T M, Katsnelson M I, Novoselov K S, Morozov S V, Zhukov A A, Schedin F, Hill E W, Geim A K. Effect of a high- $\kappa$ environment on charge carrier mobility in graphene. Phys Rev Lett 102(20): 206603 (2009)

[17] Liao L, Bai J W, Qu Y Q, Huang Y, Duan X F. Single-layer graphene on $\mathrm{Al}_{2} \mathrm{O}_{3} / \mathrm{Si}$ substrate: Better contrast and higher performance of graphene transistors. Nanotechnology 21(1): 015705 (2010)

[18] Lafkioti M, Krauss B, Lohmann T, Zschieschang U, Klauk H, Klitzing K V, Smet J H. Graphene on a hydrophobic substrate: Doping reduction and hysteresis suppression under ambient conditions. Nano Lett 10(4): 1149-1153 (2010)

[19] Leob L B. The basic mechanisms of static electrification. Science 102(2658): 573-576 (1945)

[20] Diaz A F, Felix-Navarro R M. A semi-quantitative triboelectric series for polymeric materials: The influence of chemical structure and properties. J Electrostat 62(4): 277-290 (2004)

[21] Bart S F, Mehregany M, Tavrow L S, Lang J H, Senturia $\mathrm{S}$ D. Electric micromotor dynamics. IEEE Trans Electron Devices 39(3): 566-575 (1992)

[22] Beerschwinger U, Reuben R L, Yang S J. Frictional study of micromotor bearings. Sens Actuators A: Phys 63(3): 229-241 (1997) 
[23] He F, Xie G X, Luo J B. Electrical bearing failures in electric vehicles. Friction 8(1): 4-28 (2020)

[24] Dayo A, Alnasrallah W, Krim J. Superconductivity-dependent sliding friction. Phys Rev Lett 80(8): 1690-1693 (1998)

[25] Kisiel M, Gnecco E, Gysin U, Marot L, Rast S, Meyer E. Suppression of electronic friction on $\mathrm{Nb}$ films in the superconducting state. Nat Mater 10(2): 119-122 (2011)

[26] Park J Y, Salmeron M. Fundamental aspects of energy dissipation in friction. Chem Rev 114(1): 677-711 (2014)

[27] Kim J H, Fu D Y, Kwon S, Liu K, Wu J Q, Park J Y. Crossing thermal lubricity and electronic effects in friction: Vanadium dioxide under the metal-insulator transition. Adv Mater Interfaces 3(2): 1500388 (2016)

[28] Pease R S. Crystal structure of boron nitride. Nature 165(4201): 722-723 (1950)

[29] Golberg D, Bando Y, Huang Y, Terao T, Mitome M, Tang C C, Zhi C Y. Boron nitride nanotubes and nanosheets. ACS Nano 4(6): 2979-2993 (2010)

[30] Pacilé D, Meyer J C, Girit Ç Ö, Zettl A. The two-dimensional phase of boron nitride: Few-atomic-layer sheets and suspended membranes. Appl Phys Lett 92(13): 133107 (2008)

[31] Golberg D, Bando Y, Tang C C, Zhi C Y. Boron nitride nanotubes. Adv Mater 19(18): 2413-2432 (2007)

[32] Paine R T, Narula C K. Synthetic routes to boron nitride. Chem Rev 90(1): 73-91 (1990)

[33] Chen Y, Zou J, Campbell S J, Le Caer G. Boron nitride nanotubes: Pronounced resistance to oxidation. Appl Phys Lett 84(13): 2430 (2004)

[34] Kubota Y, Watanabe K, Tsuda O, Taniguchi T. Deep ultraviolet light-emitting hexagonal boron nitride synthesized at atmospheric pressure. Science 317(5840): 932-934 (2007)

[35] Watanabe K, Taniguchi $T$, Niiyama $T$, Miya $K$, Taniguchi M. Far-ultraviolet plane-emission handheld device based on hexagonal boron nitride. Nat Photonics 3(10): 591-594 (2009)

[36] Lui C H, Liu L, Mak K F, Flynn G W, Heinz T F. Ultraflat graphene. Nature 462(7271): 339-341 (2009)

[37] Dean C R, Young A F, Meric I, Lee C, Wang L, Sorgenfrei S, Watanabe K, Taniguchi T, Kim P, Shepard K L, Hone J. Boron nitride substrates for high-quality graphene electronics. Nat Nanotechnol 5(10): 722-726 (2010)

[38] Qi Y B, Park J Y, Hendriksen B L M, Ogletree D F, Salmeron M. Electronic contribution to friction on GaAs: An atomic force microscope study. Phys Rev B 77(18): 184105 (2008)

[39] He F, Yang X, Bian Z L, Xie G X, Guo D, Luo J B. In-plane potential gradient induces low frictional energy dissipation during the stick-slip sliding on the surfaces of 2D materials. Small 15(49): 1904613 (2019)

[40] Fann W S, Storz R, Tom H W K, Bokor J. Electron thermalization in gold. Phys Rev B 46(20): 13592 13595 (1992)

[41] Novoselov K S, Castro Neto A H. Two-dimensional crystals-based heterostructures: Materials with tailored properties. Phys Scr T146: 014006 (2012)

[42] Wagner K, Cheng P, Vezenov D. Noncontact method for calibration of lateral forces in scanning force microscopy. Langmuir 27(8): 4635-4644 (2011)

[43] Green C P, Lioe H, Cleveland J P, Proksch R, Mulvaney P, Sader J E. Normal and torsional spring constants of atomic force microscope cantilevers. Rev Sci Instrum 75(6): 1988-1996 (2004)

[44] Riedo E, Lévy F, Brune H. Kinetics of capillary condensation in nanoscopic sliding friction. Phys Rev Lett 88(18): 185505 (2002)

[45] Popov V L. Contact Mechanics and Friction. Berlin, Heidelberg (Germany): Springer, 2010.

[46] Carpick R W, Ogletree D F, Salmeron M. A general equation for fitting contact area and friction vs load measurements. J Colloid Interface Sci 211(2): 395-400 (1999)

[47] Falin A, Cai Q R, Santos E J G, Scullion D, Qian D, Zhang R, Yang Z, Huang S M, Watanabe K, Taniguchi T, et al. Mechanical properties of atomically thin boron nitride and the role of interlayer interactions. Nat Commun 8(1): 15815 (2017)

[48] Tayebi N, Zhang Y G, Chen R J, Tran Q, Chen R, Nishi Y, Ma Q, Rao V. An ultraclean tip-wear reduction scheme for ultrahigh density scanning probe-based data storage. ACS Nano 4(10): 5713-5720 (2010)

[49] Cramer T, Zerbetto F, García R. Molecular mechanism of water bridge buildup: Field-induced formation of nanoscale menisci. Langmuir 24(12): 6116-6120 (2008)

[50] Gómez-Moñivas S, Sáenz J J, Calleja M, García R. Field-induced formation of nanometer-sized water bridges. Phys Rev Lett 91(5): 056101 (2003)

[51] Kurtin S, McGill T C, Mead C A. Fundamental transition in the electronic nature of solids. Phys Rev Lett 22(26): 1433 (1969)

[52] Lowell J, Rose-Innes A C. Contact electrification. $A d v$ Phys 29(6): 947-1023 (1980)

[53] Wåhlin A, Bäckström G. Sliding electrification of Teflon by metals. J Appl Phys 45(5): 2058-2064 (1974)

[54] Arridge R G C. The static electrification of nylon 66. $\mathrm{Br}$ J Appl Phys 18(9): 1311-1316 (1967)

[55] Rozhok S, Sun P, Piner R, Lieberman M, Mirkin C A. AFM study of water meniscus formation between an AFM tip and $\mathrm{NaCl}$ substrate. $J$ Phys Chem $B$ 108(23): 7814-7819 (2004) 


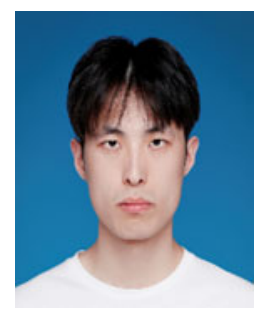

Kemeng YU. He obtained his B.Eng. degree in Hunan Institute of Technology, China (2018). He is now a master candidate in Donghua University, China. His current research interests mainly focus on nanofriction in 2D materials.

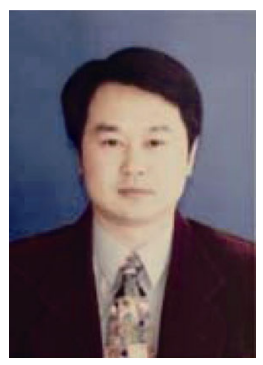

Kun ZOU. He received his B.Eng. and M.Eng. degrees in mechanical engineering from Wuhan University of Technology. He received his Ph.D. degree from Tsinghua University in

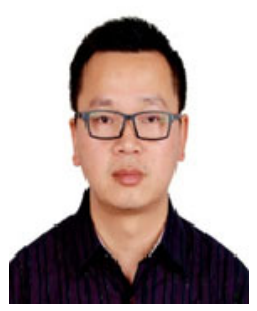

Yitian PENG. He received his Ph.D. degree from the State key laboratory of Tribology in Tsinghua University, China (2007). Now, he is a full professor at the College of Mechanical Engineering, Donghua University. He is a member of the Chinese Tribology Institute in the Chinese Mechanical Engineering Society and Micro-nano Manufacturing Institute of Chinese Micro-nano

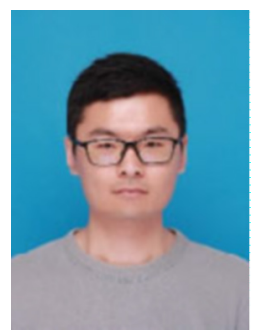

Haojie LANG. He received his Ph.D. degree in 2020 from Donghua University, Shanghai, China. He is
1999. He is an associate professor at the College of Mechanical Engineering, Donghua University, Shanghai, China. His research interests include nanomaterials, nanofriction, nanosensors, and their industrial application.

Society. He was in charge of more than 20 municipal and national projects, among which four projects were funded by the National Natural Science Foundation of China. He has published 70 peerrefereed papers among which 54 papers were published in SCI journals. The papers have been cited 705 times His current research interests focus on tribology, micro/nano fabrication, and surface manufacture.

now working as a lecturer at the College of Mechanical Engineering, Donghua University. His research focuses on nanoscale friction of 2D materials. 